\title{
Tomonaga-Luttinger liquid in the edge channels of a quantum spin Hall insulator
}

\author{
R. Stühler, ${ }^{1}$ F. Reis,${ }^{1}$ T. Müller, ${ }^{2}$ T. Helbig, ${ }^{2}$ T. Schwemmer, ${ }^{2}$ R. Thomale, ${ }^{2}$ J. Schäfer, ${ }^{1, *}$ and R. Claessen ${ }^{1}$ \\ ${ }^{1}$ Physikalisches Institut and Röntgen Research Center for Complex Material Systems, \\ Universität Würzburg, D-97074 Würzburg, Germany \\ ${ }^{2}$ Institut für Theoretische Physik und Astrophysik, \\ Universität Würzburg, D-97074 Würzburg, Germany
}

(Dated: January 21, 2019)

\begin{abstract}
Topological quantum matter is characterized by non-trivial global invariants of the bulk which induce gapless electronic states at its boundaries. A case in point are two-dimensional topological insulators (2D-TI) which host one-dimensional (1D) conducting helical edge states protected by time-reversal symmetry (TRS) against singleparticle backscattering (SPB). However, as twoparticle scattering is not forbidden by TRS [1], the existence of electronic interactions at the edge and their notoriously strong impact on 1D states may lead to an intriguing interplay between topology and electronic correlations. In particular, it is directly relevant to the question in which parameter regime the quantum spin Hall effect (QSHE) expected for 2D-TIs becomes obscured by these correlation effects that prevail at low temperatures [2]. Here we study the problem on bismuthene on $\mathrm{SiC}(0001)$ which has recently been synthesized and proposed to be a candidate material for a room-temperature QSHE [3]. By utilizing the accessibility of this monolayer-substrate system on atomic length scales by scanning tunneling microscopy/spectroscopy (STM/STS) we observe metallic edge channels which display 1D electronic correlation effects. Specifically, we prove the correspondence with a TomonagaLuttinger liquid (TLL), and, based on the observed universal scaling of the differential tunneling conductivity $(d I / d V)$, we derive a TLL parameter $K$ reflecting intermediate electronic interaction strength in the edge states of bismuthene. This establishes the first spectroscopic identification of 1D electronic correlation effects in the topological edge states of a 2D-TI.
\end{abstract}

The topological protection of the 1D metallic edge channels in 2D-TIs against elastic SPB by TRS [4, 5] leads to quantized, i.e. dissipationless transport which is reflected in the QSHE. Moreover, the property of spin-momentum locking renders 2D-TIs promising candidate materials for applications in spintronics. To date, the QSHE has only been measured in three material systems that are all characterized by small bandgaps $\left(E_{\text {gap }} \leq 55 \mathrm{meV}\right)$ of which the quantum well $(\mathrm{QW})$ structures of three-dimensional semiconductors, such as

* e-mail: joerg.schaefer@physik.uni-wuerzburg.de
$\mathrm{HgTe} / \mathrm{CdTe}$ [6] and InAs/GaSb [7, constitute the most prominent realizations. Recently, the QSHE effect has been reported to be observed up $100 \mathrm{~K}$ in monolayer crystals of $\mathrm{WTe}_{2}, 8,9$. While most of these experiments can be well-understood within topological band theory of non-interacting electrons, the deviations from a sharply quantized conductance $\left(2 e^{2} / h\right)$ seen at very low temperatures in InAs/GaSb QW transport measurements have been attributed to the relevance of electronic interactions 2. In fact, in any real 2D-TI such interactions are inevitably present and will play a non-negligible role for the 1D edge states which are consequently expected to constitute a helical TLL. 1, 10, A direct spectroscopic identification of this correlated many-body state in the edge states of a 2D-TI, however, has remained lacking so far.

Bismuthene, i.e. a 2D monolayer of $\mathrm{Bi}$ atoms epitaxially grown on a semiconducting $\mathrm{SiC}(0001)$ substrate, turns out to be a particularly well-suited system for such studies. Here the strong atomic spin-orbit coupling in the $\mathrm{Bi}$ atoms conspires with their honeycomb arrangement and covalent coupling to the substrate to drive the system into a non-trivial topology [3, 11]. Angle-resolved photoelectron spectroscopy as well as STM/STS found excellent agreement with the calculated non-trivial topological band structure [3] and confirmed a sizable fundamental band gap $(\Delta \sim 0.8 \mathrm{eV})$, potentially allowing the persistence of the QSHE up to room-temperature and beyond. Moreover, STM/STS measurements clearly indicate the existence of conducting edge channels which due to the large bulk band gap display an extremely small exponential decay length $\xi$ into the insulating $2 \mathrm{D}$ bulk of only $4.1 \AA[3]$.

The near-to-perfect spatial $1 \mathrm{D}$ confinement of the metallic edge states is ideally matched to the atomic resolution of STM/STS, as illustrated Fig. 1. While with constant current STM we are able to explore the surface topography, STS allows us to spatially map the local electronic density of states (LDOS). Accordingly, the STM topography map in Fig. 1. (a) demonstrates the successful synthesis of bismuthene with its planar atomic honeycomb structure. While bismuthene covers $\mathrm{SiC}$ smoothly within substrate terraces, the monolayer film is truncated at $\mathrm{SiC}$ zigzag terrace steps. Due to the $\sqrt{3}-\mathrm{R} 30^{\circ}$ superstructure of the Bi honeycomb lattice with respect to the substrate, this translates into well-ordered armchair edges of the bismuthene layer (see Fig. 1(a) and Fig. S1). We note that the periodic spacing between the bright 

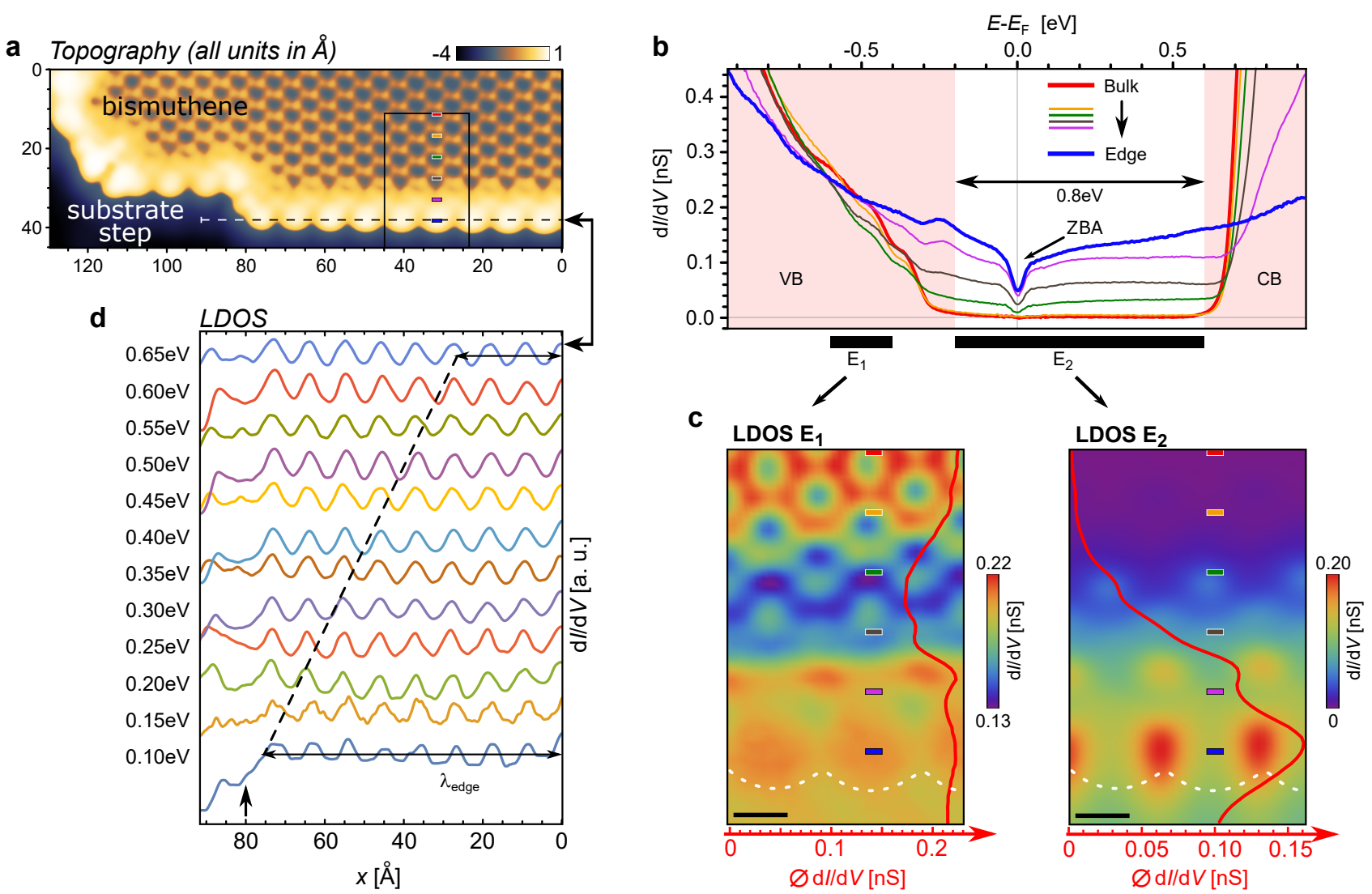

FIG. 1. Strongly confined 1D metallic channels at bismuthene armchair edges. a, Constant current image (topography) of a bismuthene armchair edge terminated by a $\mathrm{SiC}$ terrace step $\left(T=4.4 \mathrm{~K}, V_{\text {set }}=-1.1 \mathrm{~V}, I_{\text {set }}=100 \mathrm{pA}\right)$. b, $d I / d V(V)$ spectra at positions indicated by the colored marks in a and c. The edge LDOS is suppressed $\pm 100 \mathrm{meV}$ around $E_{\mathrm{F}}$ (ZBA). c, (left) Spatially resolved LDOS-map averaged over an energy range $E_{1}:-0.6 \mathrm{eV} \leq E-E_{\mathrm{F}} \leq-0.4 \mathrm{eV}$ in the occupied states. The bismuthene bulk LDOS can clearly be seen as honeycombs. (right) Spatially resolved LDOS map averaged over the bulk energy gap $E_{2}:-0.2 \mathrm{eV} \leq E-E_{\mathrm{F}} \leq 0.6 \mathrm{eV}$. The bulk states disappear and only a $\sim 1.5$ nm wide metallic channel remains at the edge that extents along the terrace step. White dashed lines: Edge in the topography. Red curves: Spatially and energetically averaged differential conductivities perpendicular to the edge. Within the bandgap the averaged differential conductivity confirms the exponential decay $(\xi=4.1 \AA)$ of the edge LDOS towards the bulk. The scale bar corresponds to $5 \AA$. (See movie in supplementary information.) d, Waterfall plot of LDOS line profiles at different energies measured along the dashed line in a. The double arrow marks the electron wavelength $\lambda_{\text {edge }}$ according to DFT calculations. 3] The black arrow indicates the position of the kink in the bismuthene edge along the $\mathrm{x}$-direction.

white, oval edge features in Fig. 1(a) is in agreement with theoretical expectations for armchair edges and indicates the accumulation of charge density at the edge.

This assumption is supported by the STS LDOS data. While the differential conductivity measured well inside the bulk material (Fig. 1(b), red curve) is dominated by the bulk bismuthene band gap, it becomes continuously filled as the tunneling tip moves towards the film edge (thin curves in Fig. 1(b)). Eventually, directly at the edge (blue curve in Fig (b)) the differential conductivity indicates a nearly constant LDOS, except for the apparent dip around $E_{F}$ indicated by 'ZBA' and discussed further below. Spatially resolved $d I / d V(V)$-maps taken parallel to the bismuthene edge (Fig. 1(c)) further elucidate the $1 \mathrm{D}$ character of the edge LDOS. Within the occupied states, e.g., in the energy range $E_{1}$, the bismuthene bulk LDOS can be seen clearly. Evidently the edge LDOS has already set in. In contrast, inside the bulk energy gap $E_{2}$ the LDOS is confined solely to the geometrical edge, defining a highly constricted 1D metallic channel spreading along the entire extent of the $\mathrm{SiC}$ terrace step edge $(\sim 10 \mathrm{~nm}-200 \mathrm{~nm})$. (See movie in supplementary information for full evolution of the edge LDOS with energy.)

We notice that the edge in Fig. 11(a) encompasses a kink in the substrate step. For an ordinary $1 \mathrm{D}$ electron wave one would expect a partial reflection off the kink causing a quasi-particle interference with the incident wave. Such emerging Friedel oscillations would scale with the energydependent electron wavelength $\lambda_{\text {edge }}$ defined by the band dispersion. 3] Eventually, the electrons in an ordinary $1 \mathrm{D}$ conductor may even become localized depending on the strength of the potential scatterer [12. However, in our case the periodicity seen in the edge LDOS is exclusively caused by the topographic, i.e. atomic modulation 
a

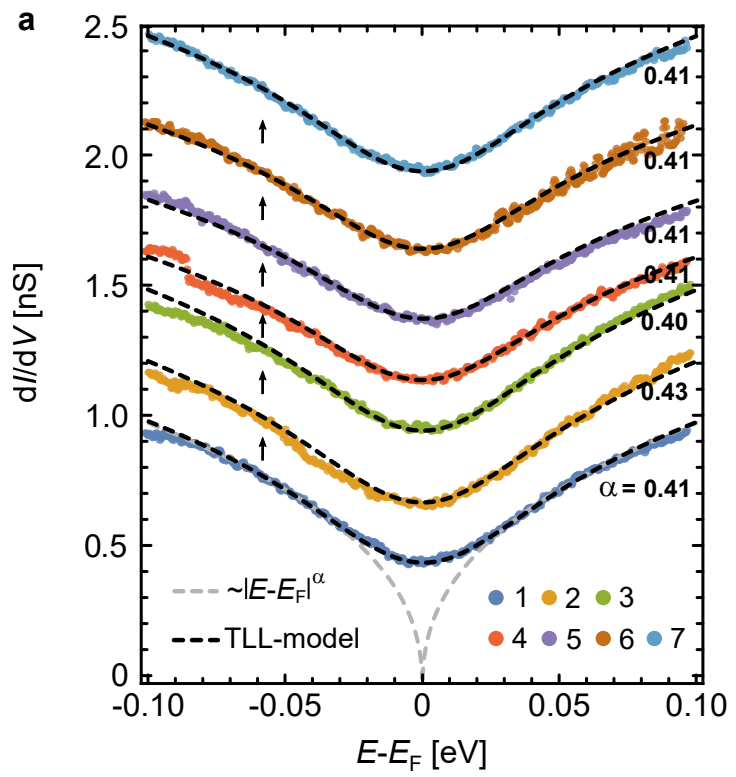

b

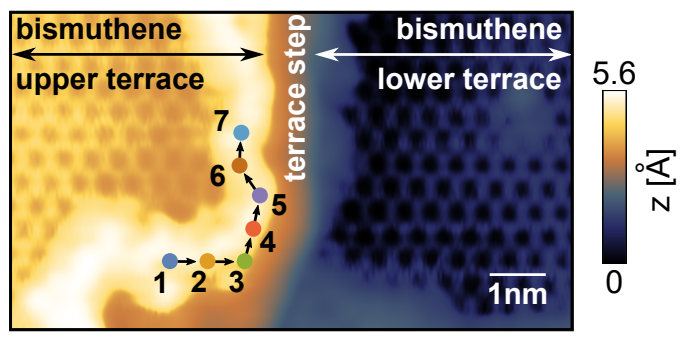

FIG. 2. Power-law suppression of the ZBA. a, Waterfall plot of single-point STS spectra along the path in b $(T=95 \mathrm{~K}$, $\left.V_{\text {set }}=-0.1 \mathrm{~V}, I_{\text {set }}=300 \mathrm{pA}\right)$. Spectra $2-7$ are offset by multiples of $0.25 \mathrm{nS}$. The ZBA follows a power-law $\sim \mid E-$ $\left.E_{\mathrm{F}}\right|^{\alpha}$ (gray dotted line). The fitted power-law exponent $\alpha$ is listed on the right margin. Black dashed line: TLL model $\rho_{\text {TLL }}$ according to Eq. (1) (taking into account thermal and instrumental broadening) shows excellent agreement with the data over the entire energy range $\pm 100 \mathrm{meV}$ around $E_{\mathrm{F}}$. b, Constant current image of bismuthene at a $\mathrm{SiC}$ step $(T=$ $\left.95 \mathrm{~K}, V_{\text {set }}=-1.0 \mathrm{~V}, I_{\text {set }}=300 \mathrm{pA}\right)$. STS spectra are marked with colored dots from $1-7$ along a path at the upper terrace that encounters a kink at the edge.

of the armchair edge, irrespective of electron energy as can clearly be seen in Fig. 1(d). Most importantly, no energy-dependent Friedel oscillations are observed, consistent with the topological protection of the helical edge states in a 2D-TI against SPB. Consequently, the effective mean free path of the edge channels is extraordinarily large, as kinks and (non-magnetic) impurity potentials do not act as scattering centers 13 .

The LDOS of a (metallic) Fermi liquid is expected to be finite and nearly constant around the Fermi level. In contrast, our edge state spectra display a clearly detectable dip at $E_{\mathrm{F}}$ which in the following will be referred to as zero-bias anomaly (ZBA). In an energy window of $\approx \pm 100 \mathrm{meV}$ around $E_{\mathrm{F}}$ it displays particle-hole symmet- ric behavior following (not too close to $E_{\mathrm{F}}$ ) a power-law $\propto\left|E-E_{\mathrm{F}}\right|^{\alpha}$ with $\alpha \sim 0.41$ as high-resolution single-point STS reveals in Fig.2(a). Interestingly, spectral shape and power law exponent $\alpha$ of the ZBA persist along the entire length of the 1D edge channel and do not even change as one follows the LDOS around a strongly kinky section of the edge (see Fig. 2(b) for measurement path), reminiscent of the absence of SPB at the edge kinks.

The ZBA also features a pronounced temperature dependence, see Fig. 3(a). As the temperature increases from $4.4 \mathrm{~K}$ to $110 \mathrm{~K}$ the zero energy dip gets continuously filled. For further quantitative analysis Fig. 3(b) shows the normalized zero-bias differential conductivity $d I / d V(V=0)$ as a function of temperature on a double logarithmic scale. It is apparent that for $T>10 \mathrm{~K}$ the zero-bias differential conductivity again follows a powerlaw $d I / d V(V=0) \sim T^{\alpha}$, with the same exponent $\alpha \sim 0.41$ already observed in the energy dependence. For $T<10 \mathrm{~K}$ deviations from the bare power-law are due to instrumental broadening. The finite bias differential conductivity, on the other hand, and especially the energy power law remains constant as a function of temperature (see Fig $3(\mathrm{c})$ ).

It is well-established that electronic interactions play a crucial role in 1D metallic systems. As described by TLL theory, the Fermi liquid description breaks down in 1D and the physics is dominated by collective bosonic excitations 14, 15. Signatures of ordinary (spinful) TLLs have been observed, e.g., in semiconducting GaAs quantum wires [16], metallic single wall carbon nanotubes [17], in polymer nanofibers [18, and atomic chains of gold atoms on Ge surfaces [19. TLL behavior has also been identified in the chiral edge states of fractional quantum Hall systems [20]. A special case are the spin-momentumlocked edge states in a 2D-TI which form a helical TLL as long as the electronic interaction is weaker than the band gap that protects them from the $2 \mathrm{D}$ bulk states 21. However, one common characteristic inherent to all (spinful and helical) TLLs is a power-law behavior of their single-particle spectrum, $\rho \sim\left|E-E_{\mathrm{F}}\right|^{\alpha}$ for $\left|E-E_{\mathrm{F}}\right| \gg k_{B} T[22$, directly accessible by experimental electron removal and addition spectroscopies, such as STS [23]. In fact, the energy dependence emerges from an even more fundamental property of TLLs, namely the universal scaling in both energy and temperature which is captured by the expression [17]:

$\rho_{\mathrm{TLL}}(\epsilon, T) \propto T^{\alpha} \cosh \left(\frac{\epsilon}{2 k_{B} T}\right)\left|\Gamma\left(\frac{1+\alpha}{2}+i \frac{\epsilon}{2 \pi k_{B} T}\right)\right|^{2}$.

This inherently generates also a temperature power-law dependence of the zero energy spectral weight with the same exponent as in energy. Comparing with our experimental STS data we find excellent agreement with the theoretical $\rho_{\text {TLL }}$ over the entire energy and temperature range as seen in Fig. 2(a) and Fig. 3(a)(black curves), suggesting that the $\mathrm{ZBA}$ observed in the edge states is indeed due to strong $1 \mathrm{D}$ electron corrrelations. 
a

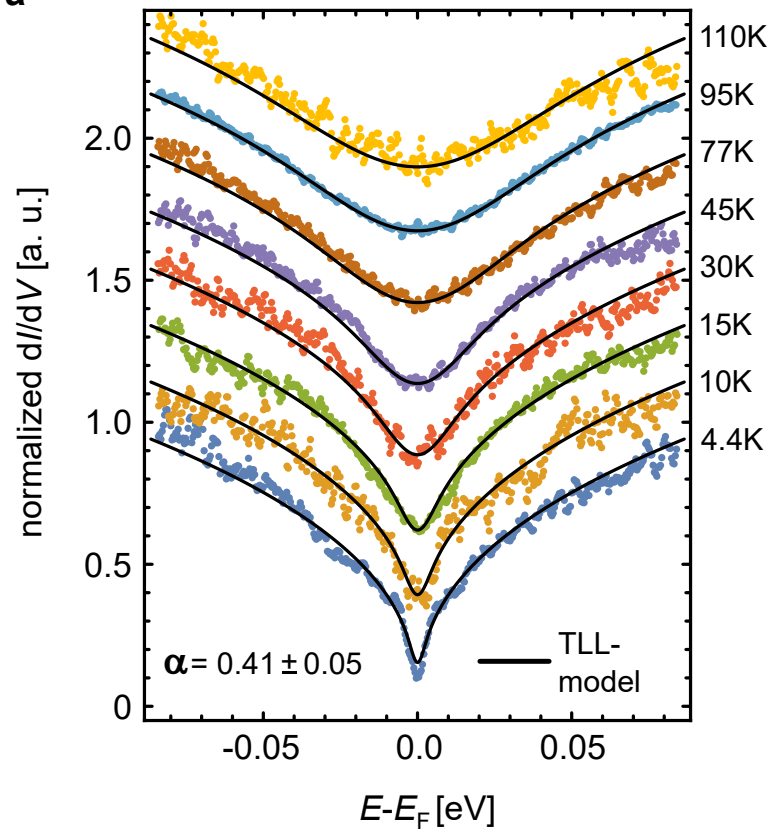

b

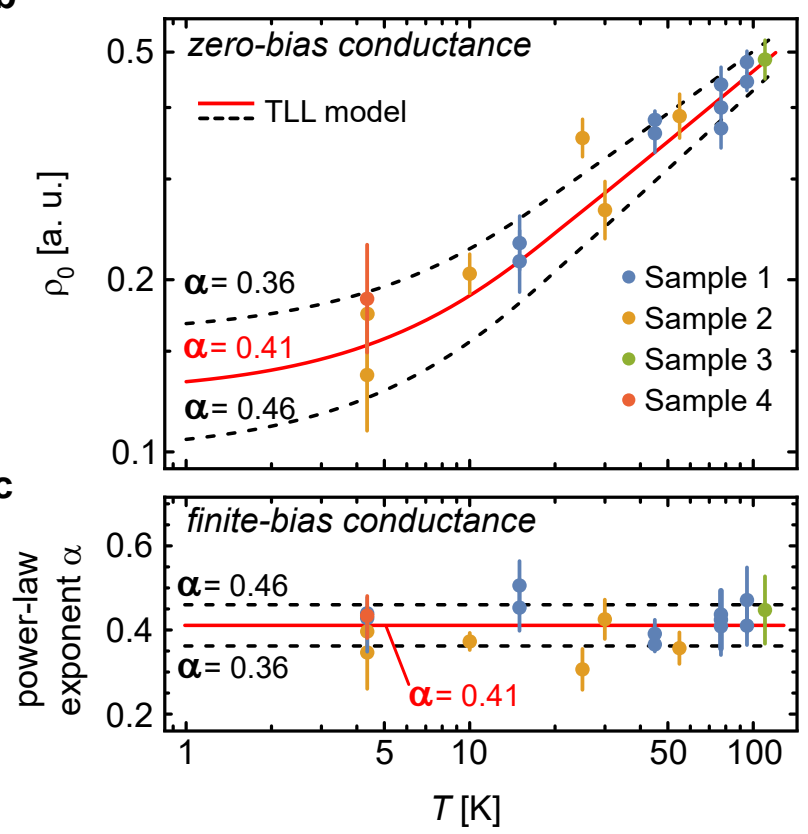

FIG. 3. Temperature dependence of the ZBA. a, Waterfall plot of single-point STS spectra for different temperatures. Spectra from $10 \mathrm{~K}-110 \mathrm{~K}$ are offset by multiples of 0.2 . Towards higher temperatures the ZBA becomes continuously filled. Black lines: all spectra are described by Eq. (1) (taking into account thermal and instrumental broadening). The power-law exponent $\alpha$ is the only fit parameter for the TLL model. $\mathbf{b}$, Double-logarithmic plot of the zero-bias conductance $\rho_{0}(T)$ as a function of temperature. Each data point and error bar reflects the evaluation of $\sim 50$ spectra. Red and black dashed lines: for $T>10 \mathrm{~K} \rho_{0}(T)$ clearly follows a power-law $\rho_{0} \sim T^{\alpha}$. Deviations from the bare power-law for $T<10 \mathrm{~K}$ are due to instrumental broadening. c, Power-law exponent $\alpha$ for the finite-bias conductance $\rho(T) \sim\left|E-E_{\mathrm{F}}\right|^{\alpha}$ as a function of temperature. Each data point and error bar reflects the evaluation of $\sim 50$ spectra. Red and black dashed lines: the energy power law remains constant as a function of temperature.

However, the TLL interpretation has to be discriminated against other possible origins of a ZBA. One scenario is the Efros-Shklovskii pseudogap which can arise from the interplay of disorder and Coulomb interaction in low-dimensional metallic systems. It is characterized by an exponential suppression of the LDOS 24. As the observed ZBA in bismuthene follows a power-law instead, the disorder-induced pseudogap can be safely excluded (see also Fig. S5 for a direct comparison). Another mechanism that can lead to a suppression of the low-energy tunneling is the dynamical Coulomb blockade (DCB) [25, 26] caused by the interplay between charge storage energy in and charge dissipation from a nanostructure (here the $1 \mathrm{D}$ edge). Within environment-quantum fluctuation or $P(E)$-theory this can be treated by modeling the STS tunneling junction as a capacitance $C$ parallel to a resistance $R$. For $\left|E-E_{\mathrm{F}}\right| \ll E_{c}=e^{2} / 2 C$ and at zero temperature a power-law behavior is predicted according to $d I / d V(E) \sim\left|E-E_{\mathrm{F}}\right|^{2 / g}$ with the exponent $2 / g=2 R / R_{K}$ [26], $R_{K}=h / e^{2}$ being the resistance quantum. We have measured the ZBA on four different samples, on bismuthene domains with differing sizes, and with widely varied set-point parameters $I_{\text {set }}$ and $V_{\text {set }}$ (see Fig. S4), where the local tunnel junction parameters $C$ and $R$ should widely differ accordingly. Yet, in all cases we observe the same power law exponent $\alpha=0.41 \pm 0.05$, at variance with the DCB picture. Finally, universal scaling would not be observed in the DCB scenario for the same locality reasons [27.

On the other hand, universal scaling can be directly tested for our tunneling spectra. In Fig. 4 the experimental $d I / d V$ data are normalized to $T^{0.4 \mathrm{I}}$ and plotted versus $e V / k_{\mathrm{B}} T$. Unambiguously all spectra collapse onto a single universal curve (red curve) as predicted for a TLL according to Eq. (1] [17, 19. Our observation leads to the conclusion that the $\mathrm{ZBA}$ is a result of intrinsic properties of the $1 \mathrm{D}$ edge channel and cannot arise from the DCB scenario. Ultimately, our observations provide critical proof to render bismuthene the first 2D-TI system for which a TLL behavior has been established and studied on the atomic scale. Moreover, the absence of Friedel oscillations is in agreement with the TLL in bismuthene being helical.

Within TLL theory the strength of the Coulomb interaction $W$ between the electrons in the 1D edge channel can be expressed by the parameter $K \approx$ $\left[1+W / \pi \hbar v_{\mathrm{F}}\right]^{-1 / 2}$, where $v_{\mathrm{F}}$ is the Fermi velocity. For non-interacting electrons $K=1$. Moderate to strong electronic interactions result in two-particle scattering in the edge channels of the 2D-TI (see Ref. [1, 10]), rendering $K$ essential for the interpretation of transport mea- 


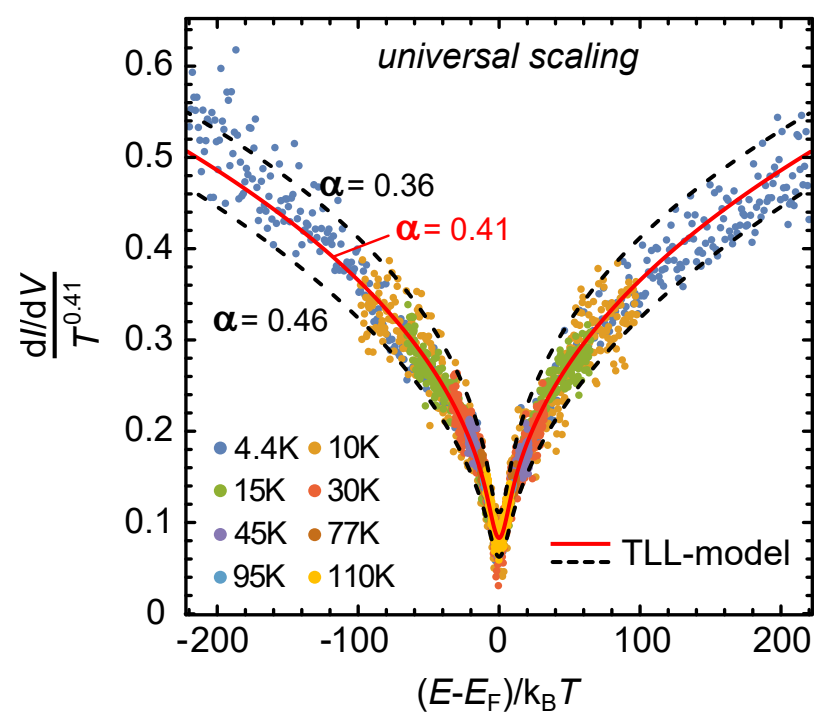

FIG. 4. Universal scaling of the ZBA as a hallmark of a TLL. On properly rescaled axes the single-point STS spectra of Fig. 3(a) collapse onto a single universal curve predicted as a hallmark for tunneling into a TLL. Red curve: $\rho_{\text {TLL }}$ according to Eq. (1) (taking into account thermal and instrumental broadening) with exponent $\alpha=0.41$. Black dashed curve: for \pm 0.05 variation of $\alpha$ to test statistical confidence (for a log-log plot see the supplementary information Fig. S3).

surements on 2D-TIs. To date, only few experimental studies have addressed the effect of electronic correlations in the edge channels. A notable exception is a recent transport study of InAs/GaSb QWs which reported experimental evidence for a helical TLL [2]. However, the extraction of $K$ from transport data is ambiguous, because the resulting values vary widely depending on the underlying theory ( $K=0.22[2]$ vs. $K=0.8[28]$ ). Many authors propose a more direct determination of $K$ by local spectroscopy (i.e. STS) of the electronic spectral function [17, 19, 23]. Accordingly, for a helical TLL the characteristic power-law exponent of the generic spectrum (1) is directly related to $K$ via $\alpha=\frac{1}{2}(K+1 / K-2)$ (note the prefactor $\frac{1}{2}$ which for a spinful TLL would be $\frac{1}{4}$ [22]). The experimentally determined exponent $\alpha$ thus translates into the helical TLL parameter $K=0.42 \pm 0.05$ for the bismuthene armchair edge channels.

Qualitatively, the effective electronic interaction strength is expected to be enhanced (reduced) with decreasing (increasing) Fermi velocity $v_{\mathrm{F}}$ and confinement length $\xi$ of the 1D edge channels. For example, for $\mathrm{HgTe} / \mathrm{CdTe} \mathrm{QWs}\left(v_{\mathrm{F}}=5.3 \times 10^{5} \mathrm{~m} / \mathrm{s}, \xi=30 \mathrm{~nm}\right)$ theoretical estimates yield $K \approx 0.8$ (Ref. [29]), placing this system in the weakly to nearly non-interacting regime. In contrast, for bismuthene the key parameters are $v_{\mathrm{F}}=5.5 \times 10^{5} \mathrm{~m} / \mathrm{s}$ and $\xi \approx 0.4 \mathrm{~nm} 3$. Such exceptionally narrow confinement length $\xi$ (2 orders of magnitude less than in $\mathrm{HgTe} / \mathrm{CdTe} \mathrm{QWs}$ ) is driven by the large bulk gap of $\Delta \sim 0.8 \mathrm{eV}$ in bismuthene $\left(\xi \approx \hbar v_{F} / \Delta\right)$. This sug- gests a substantially stronger interaction, which is nicely confirmed by our experimental value $K=0.42$. It is further underpinned by a theoretical model for bismuthene which results in $K \approx 0.5 \pm 0.1$ (see supplementary information).

Bismuthene on $\mathrm{SiC}$ is thus the first 2D-TI candidate in which the spectral properties of a (helical) TLL have been verified in its $1 \mathrm{D}$ edge states. The experimentally determined helical TLL parameter $K$ indicates that the edge channels - while topologically protected against SPB - display pronounced two-particle scattering. With regard to the QSHE, bismuthene is thus a model system to study in which way the conductance quantization is affected by these correlation effects.

Methods Bismuthene was grown on n-doped 4H-SiC(0001) substrates with a resistivity of $0.01 \Omega \mathrm{cm}-0.03 \Omega \mathrm{cm}$ at roomtemperature. The dopant concentration of the substrate is $5 \times 10^{18} \mathrm{~cm}^{-3}-1 \times 10^{19} \mathrm{~cm}^{-3}$. The STM/STS measurements have been performed with a commercial low-temperature STM from Scienta Omicron GmbH under UHV conditions ( $p_{\text {base }}=2 \times 10^{-11} \mathrm{mbar}$ ). Topographic STM images are recorded as constant current images. After stabilizing the tip at a voltage and current set-point $V_{\text {set }}$ and $I_{\text {set }}$, respectively, the feedback loop is opened and STS spectra are obtained making additional use of a standard lock-in technique with a modulation frequency of $787 \mathrm{~Hz}$ and modulation amplitude $V_{\mathrm{mod}}=1 \mathrm{mV}$. Because of external modulation the instrumental resolution results in an gaussian energy broadening (FWHM: $\delta E=2.5 e V_{\text {mod }}$ ) in addition to the thermal broadening caused by the Fermi-Dirac function (FWHM $=3.5 k_{B} T$ ). We only show single-point spectra and avoid averaging over various STS spectra of a finite sample area. Prior to every measurement on bismuthene we assured that the tip LDOS is metallic and to a good approximation can be considered constant in the energy range $-100 \mathrm{meV} \leq E-E_{\mathrm{F}} \leq 100 \mathrm{meV}$ by a reference measurement on a silver surface. As a result, $d I / d V(V)$ curves measured with this tip are a good measure of the sample LDOS.

Acknowledgements We thank B. Trauzettel, A. Kowalewski and J. Maciejko for useful discussions. This work was supported by the Deutsche Forschungsgemeinschaft (DFG) through the Collaborative Research Center SFB 1170 "ToCoTronics" in Würzburg, the SPP 1666 Priority Program "Topological Insulators", and by the European Research Council (ERC) through starting grant ERC-StG-Thomale336012 "Topolectrics".

Author contributions R.S. and F.R. carried out the measurements, R.S. analysed the data and made the figures. T.M., T.H., T.S, and R.T. developed the theory for the helical edge with dielectric screening. J.S. conceived the experiment. R.S. and R.C. wrote the text with input from J.S. and F.R., and all authors contributed to critical discussion of the data. 
[1] C. Xu and J. E. Moore, Phys. Rev. B 73, 045322 (2006)

[2] T. Li, P. Wang, H. Fu, L. Du, K. A. Schreiber, X. Mu, X. Liu, G. Sullivan, G. A. Csáthy, X. Lin, and R.-R. Du, Phys. Rev. Lett. 115, 136804 (2015).

[3] F. Reis, G. Li, L. Dudy, M. Bauernfeind, S. Glass, W. Hanke, R. Thomale, J. Schäfer, and R. Claessen, Science 357 (2017)

[4] C. L. Kane and E. J. Mele, Phys. Rev. Lett. 95, 226801 (2005)

[5] C. L. Kane and E. J. Mele, Phys. Rev. Lett. 95, 146802 (2005)

[6] M. König, S. Wiedmann, C. Brüne, A. Roth, H. Buhmann, L. W. Molenkamp, X.-L. Qi, and S.-C. Zhang, Science 318, 766 (2007)

[7] I. Knez, R.-R. Du, and G. Sullivan, Phys. Rev. Lett. 107, 136603 (2011)

[8] S. Wu, V. Fatemi, Q. D. Gibson, K. Watanabe, T. Taniguchi, R. J. Cava, and P. Jarillo-Herrero, Science 359, 76 (2018)

[9] S. Tang, C. Zhang, D. Wong, Z. Pedramrazi, H.-Z. Tsai, C. Jia, B. Moritz, M. Claassen, H. Ryu, S. Kahn, J. Jiang, H. Yan, M. Hashimoto, D. Lu, R. G. Moore, C.-C. Hwang, C. Hwang, Z. Hussain, Y. Chen, M. M. Ugeda, Z. Liu, X. Xie, T. P. Devereaux, M. F. Crommie, S.-K. Mo, and Z.-X. Shen, Nat Phys 13, 683 (2017)

[10] C. Wu, B. A. Bernevig, and S.-C. Zhang, Phys. Rev. Lett. 96, 106401 (2006)

[11] G. Li, W. Hanke, E. M. Hankiewicz, F. Reis, J. Schäfer, R. Claessen, C. Wu, and R. Thomale, Phys. Rev. B 98, $165146(2018)$

[12] C. Meyer, J. Klijn, M. Morgenstern, and R. Wiesendanger, Phys. Rev. Lett. 91, 076803 (2003).

[13] C. Pauly, B. Rasche, K. Koepernik, M. Liebmann, M. Pratzer, M. Richter, J. Kellner, M. Eschbach, B. Kaufmann, L. Plucinski, C. M. Schneider, M. Ruck, J. van den Brink, and M. Morgenstern, Nat Phys 11, $338(2015)$

[14] J. Voit, Phys. Rev. B 47, 6740 (1993).

[15] F. D. M. Haldane, Journal of Physics C: Solid State Physics 14, 2585 (1981)

[16] Y. Jompol, C. J. B. Ford, J. P. Griffiths, I. Farrer, G. A. C. Jones, D. Anderson, D. A. Ritchie, T. W. Silk, and A. J. Schofield, Science 325, 597 (2009)

[17] M. Bockrath, D. H. Cobden, J. Lu, A. G. Rinzler, R. E. Smalley, L. Balents, and P. L. McEuen, Nature 397, 598 (1999)

[18] A. N. Aleshin, H. J. Lee, Y. W. Park, and K. Akagi, Phys. Rev. Lett. 93, 196601 (2004)

[19] C. Blumenstein, J. Schäfer, S. Mietke, S. Meyer, A. Dollinger, M. Lochner, X. Y. Cui, L. Patthey, R. Matzdorf, and R. Claessen, Nat Phys 7, 776 (2011)

[20] A. M. Chang, L. N. Pfeiffer, and K. W. West, Phys. Rev. Lett. 77, 2538 (1996)

[21] M. Hohenadler and F. F. Assaad, Phys. Rev. B 85, 081106 (2012)

[22] B. Braunecker, C. Bena, and P. Simon, Phys. Rev. B 85, $035136(2012)$

23] S. Eggert, Phys. Rev. Lett. 84, 4413 (2000)

[24] L. Bartosch and P. Kopietz, The European Physical Journal B - Condensed Matter and Complex Systems 28, 29 (2002)
[25] A. E. Hanna and M. Tinkham, Phys. Rev. B 44, 5919 (1991).

[26] M. H. Devoret, D. Esteve, H. Grabert, G.-L. Ingold, H. Pothier, and C. Urbina, Phys. Rev. Lett. 64, 1824 (1990).

[27] F. Ming, T. S. Smith, S. Johnston, P. C. Snijders, and H. H. Weitering, PRB 97, 075403 (2018)

[28] J. I. Väyrynen, F. Geissler, and L. I. Glazman, Phys. Rev. B 93, 241301 (2016)

[29] J. C. Y. Teo and C. L. Kane, Phys. Rev. B 79, 235321 (2009) 


\section{Supplementary Information}

Tomonaga-Luttinger liquid in the edge channels of a quantum spin Hall insulator

R. Stühler, ${ }^{1}$ F. Reis, ${ }^{1}$ T. Müller, ${ }^{2}$ T. Helbig, ${ }^{2}$ T. Schwemmer, ${ }^{2}$ R. Thomale, ${ }^{2}$ J. Schäfer, ${ }^{1, *}$ and R. Claessen ${ }^{1}$

${ }^{1}$ Physikalisches Institut and Röntgen Research Center for Complex Material Systems,

Universität Würzburg, D-97074 Würzburg, Germany

${ }^{2}$ Institut für Theoretische Physik und Astrophysik,

Universität Würzburg, D-97074 Würzburg, Germany

*e-mail: joerg.schaefer@physik.uni-wuerzburg.de

(Dated: January 21, 2019) 


\section{A. Structural considerations for an bismuthene armchair edge at a SiC zigzag terrace step}

Bismuthene is synthesized on a hydrogen-etched $\mathrm{SiC}(0001)$ substrate on which it forms a $\sqrt{3} \times \sqrt{3} R 30^{\circ}$ superstructure of $\mathrm{Bi}$ atoms in honeycomb geometry with a lattice constant of $5.35 \AA$. The tensile stress of $\approx 18 \%$ compared to a buckled $\mathrm{Bi}(111)$ monolayer causes a fully planar configuration which can be seen in the scanning tunneling microscopy (STM) constant current image by the absence of intensity corrugation between Bi atoms on honeycomb sub-lattice sites $A$ and $B$. The bismuthene film covers the entire substrate smoothly and is naturally terminated by $\mathrm{SiC}$ terrace steps. The $\mathrm{SiC}$ edges which form a zigzag configuration typically have a length of several $100 \mathrm{~nm}$. Because of bismuthene forming a $\sqrt{3} \times \sqrt{3} R 30^{\circ}$ superstructure on SiC the experimentally rare situation of armchair edges is realized in this material system (Fig. S1).

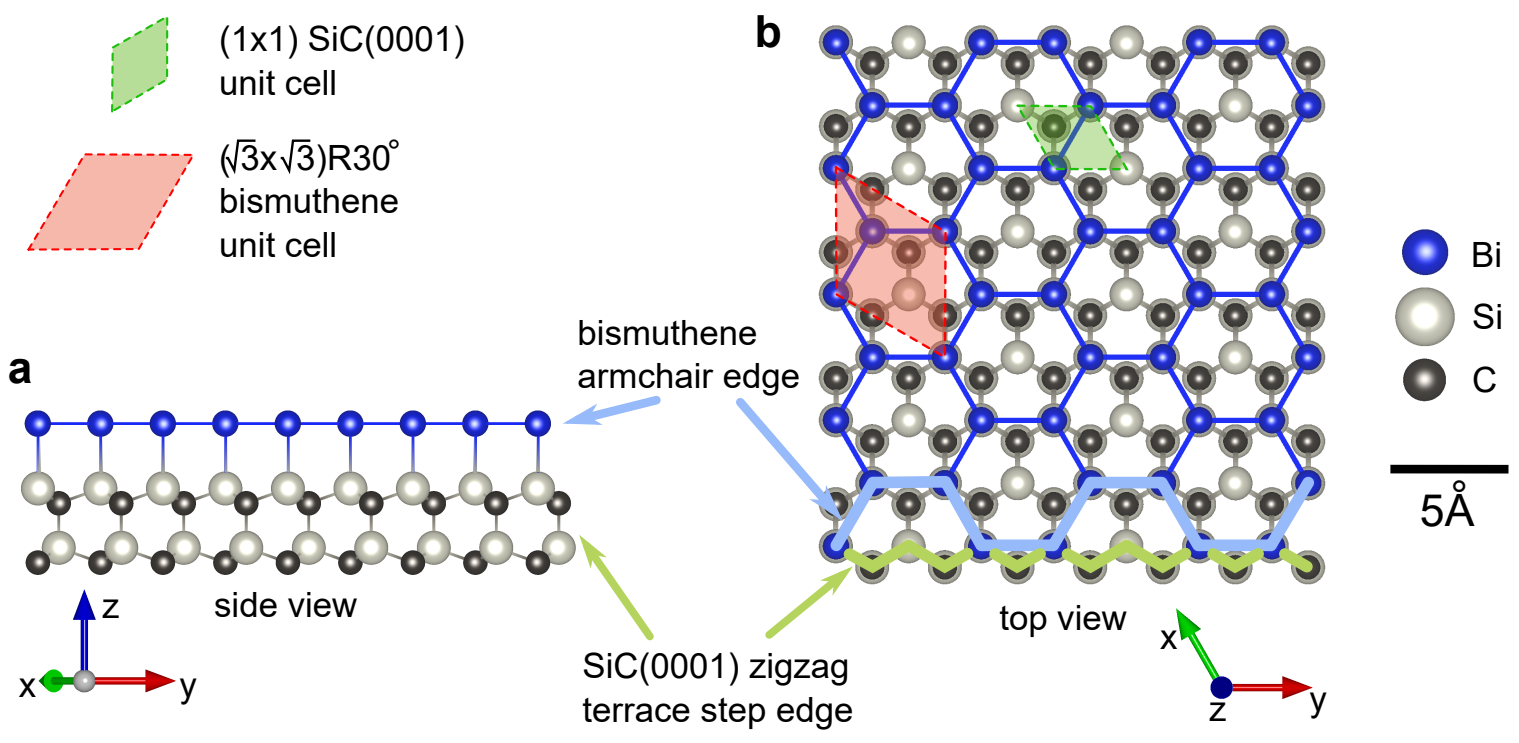

FIG. S1. Schematic overview fo bismuthene synthesized on $\mathbf{S i C}(0001)$ a, Side view of the layered system. b, Top view of the geometric placement of the bismuthene layer in a commensurate $\sqrt{3} \times \sqrt{3} R 30^{\circ}$ reconstruction. Two of three top layer $\mathrm{Si}$ atoms are binding to $\mathrm{Bi}$. The in-plane lattice constant amounts to $5.35 \AA$. Due to the underlying substrate bismuthene grows in a fully planar configuration. At $\mathrm{SiC}$ zigzag terrace step edges the bismuthene film is naturally terminated in an armchair configuration. 


\section{B. Estimate of the Luttinger parameter $K$}

Helical edge states of a 2D-TI, in principle, resemble a prototypical 1D system, as their direction of motion is limited to the direction along the edge (in our case the SiC terrace step). Topologically protected edge states decay exponentially into the bulk and are confined to the edge, where the strength of the confinement, and hence the effective width of the channel, depends on the bulk band gap and the Fermi velocity. Immediate experimental evidence for helical TLLs has so far been limited to InAs/GaSb QWs S1. Determining the interaction parameter $K$ of the Luttinger theory, which manifests itself in observable quantities (such as velocity renormalization and the power-law dependence of the LDOS upon approaching $E_{\mathrm{F}}$ ) is challenging: Values on transport measurements crucially depend on the employed theoretical phenomenology, as it is the case for InAs/GaSb QWs [S1, S2. Table I provides an overview of theoretical estimates and measurements of $K$ in realizable experimental systems.

\begin{tabular}{clll}
\hline \hline & HgTe/CdTe QWs & $\begin{array}{l}\text { InAs/GaSb QWs } \\
\text { (Transport measurements) }\end{array}$ & $\begin{array}{l}\text { bismuthene } \\
\text { (Spectroscopic measurements) }\end{array}$ \\
\hline Experiment & no data & $K=0.21$ [S1] vs. $K=0.8$ [S2] & $K=0.42 \pm 0.05$ \\
Theoretical estimate & $K=0.8$ [S3] & $K=0.22$ [S1] & $K=0.5 \pm 0.1$ \\
\hline \hline
\end{tabular}

TABLE I. Theoretical predictions and experimental data for the $K$-Parameter for experimentally realizable helical TLLs.

The more immediate approach to measure $K$ from temperature scaling behavior relies on probing the electronic spectral function via STS [S4-S6], as pursued in our work. This requires the 2D-TI system's surface to be accessible for local tunneling measurements. The characteristic spin-charge-separation which is inherent to spinful TLLs [S7] is absent for helical TLLs because spinon and holon velocities are equal as a result of spin-momentum locking. Therefore, the characteristic fingerprint of a helical TLL is the universal scaling behaviour of the LDOS as a function of energy and temperature. Inspired by Teo and Kane [S3], we derive an estimate of $K$ for the one-dimensional TI channel. We start from a low energy (long wavelength) description of the system and model the interaction by a momentum independent long-ranged Coulomb interaction.

In the low-energy limit, the Luttinger Parameter is found to be [S8]

$$
K=\left[\frac{1+y_{2}-y_{4}}{1+y_{2}+y_{4}}\right]^{1 / 2}, y_{i}=\frac{g_{i}}{2 \pi \hbar v_{F}}
$$

Here, $g_{4}\left(g_{2}\right)$ denote forward scattering amplitudes for electrons at the same (opposite) Fermi points. For the isotropic Coulomb interaction they are equal to the long wavelength component of the potential given by

$$
g_{2}=g_{4}=V(q=0)=\left[\int \mathrm{d} y V(y) e^{-i q y}\right]_{q=0}
$$

Elastic backscattering is prohibited via spin-momentum locking and preserved time-reversal symmetry. Furthermore, Umklapp processes are assumed to be negligible at generic incommensurate filling.

We model the bismuthene edge state as a three-dimensional charge distribution (see Fig. S2), which be homogeneous over the edge length $L$ in $y$-direction and the width $w$ in $z$-direction perpendicular to the bismuthene surface. The charge distribution is exponentially decaying into the bulk in $x$-direction with a localization length $\xi$ and given by

$$
\rho(x, y, z)=\frac{e}{w \xi L} \mathrm{e}^{-x / \xi} \Theta(L / 2-|y|) \Theta(z) \Theta(w-z)
$$




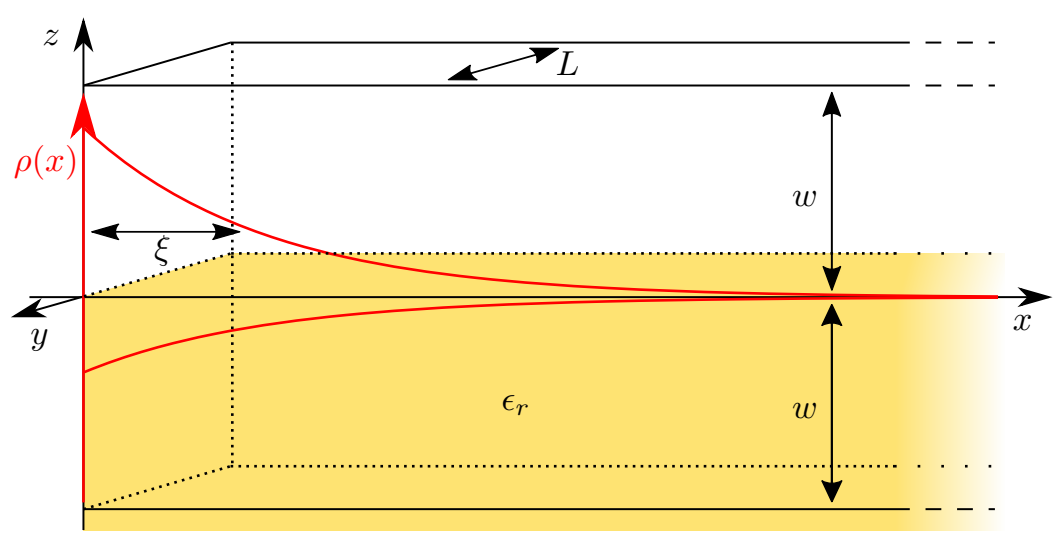

FIG. S2. Model of the charge density $\rho(x)$ of the Bismuthene edge channel on a SiC substrate (yellow) used to estimate the Luttinger parameter $K$. The charge density $\rho(x)$ is uniformly distributed over length $L$ in $y$ - and width $w$ in $z$-direction perpendicular to the interface. In $x$-direction it is subject to an exponential decay into the bismuthene bulk with a localization length $\xi$. A mirror charge with identical dimensions, opposite sign and smaller value is induced in the substrate of relative permittivity $\epsilon_{r}$.

Placing this charge distribution on top of a substrate with relative permittivity $\epsilon_{r}$ induces a mirror charge $\rho_{m}(x, y, z)=-a\left(\epsilon_{r}\right) \rho(x, y,-z)$, scaled by the factor $a\left(\epsilon_{r}\right)=\left(\epsilon_{r}-1\right) /\left(\epsilon_{r}+1\right)$. The electrostatic energy $E_{\mathrm{C}}$ of the system is composed of two parts. The first component is given by the unscreened self interaction of the charge in the edge channel. It is reduced by the second component which models the screening of the dielectric through an interaction between the channel and $\rho_{m}$. The electrostatic Coulomb energy therefore amounts to

$$
E_{\mathrm{C}}=\frac{V(q=0)}{L}=\frac{1}{4 \pi \epsilon_{0}} \int \mathrm{d}^{3} \mathbf{r} \int \mathrm{d}^{3} \mathbf{r}^{\prime}\left(\frac{\rho(\mathbf{r}) \rho\left(\mathbf{r}^{\prime}\right)}{\left|\mathbf{r}-\mathbf{r}^{\prime}\right|}+\frac{\rho(\mathbf{r}) \rho_{m}\left(\mathbf{r}^{\prime}\right)}{\left|\mathbf{r}-\mathbf{r}^{\prime}\right|}\right)
$$

As the integral in Eq. (S4) cannot be solved analytically, we will focus on two tractable limiting cases. First, we assume $\xi \ll w \ll L$, i. e. a negligible decay length. This reduces the dimensionality of the problem and results in the integral expression

$$
V_{\xi \rightarrow 0}(q=0)=\frac{e^{2}}{4 \pi \epsilon_{0}} \frac{1}{L} \int_{0}^{L} \mathrm{~d} y^{\prime} \int_{0}^{L} \mathrm{~d} y \frac{1}{w^{2}} \int_{0}^{w} \mathrm{~d} z^{\prime} \int_{0}^{w} \mathrm{~d} z\left(\frac{1}{\left|\mathbf{r}_{x=0}-\mathbf{r}_{x^{\prime}=0}^{\prime}\right|}-\frac{a\left(\epsilon_{r}\right)}{\sqrt{\left(y-y^{\prime}\right)^{2}+\left(z+z^{\prime}\right)^{2}}}\right) .
$$

We can simplify the integrated expression by expanding in $w / L$ and find

$$
V_{\xi \rightarrow 0}(q=0)=-\frac{e^{2}}{\pi \epsilon_{0}\left(\epsilon_{r}+1\right)} \ln \left(e^{-\frac{1}{2}} 2^{-\epsilon_{r}} \frac{w}{L}\right)
$$

Second, we assume the perpendicular width of the channel $w$ to be negligible, $w \ll \xi \ll L$, where Eq. (S4) reduces to

$$
V_{w \rightarrow 0}(q=0)=\frac{e^{2}}{4 \pi \epsilon_{0}} \frac{1}{L} \int_{0}^{L} \mathrm{~d} y^{\prime} \int_{0}^{L} \mathrm{~d} y \frac{1}{\xi^{2}} \int_{0}^{\infty} \mathrm{d} x^{\prime} \int_{0}^{\infty} \mathrm{d} x e^{-\frac{x+x^{\prime}}{\xi}}\left(\frac{1}{\left|\mathbf{r}_{z=0}-\mathbf{r}_{z^{\prime}=0}^{\prime}\right|}-\frac{a\left(\epsilon_{r}\right)}{\left|\mathbf{r}_{z=0}-\mathbf{r}_{z^{\prime}=0}^{\prime}\right|}\right)
$$

Here, we can expand in $\xi / L$ and find

$$
V_{w \rightarrow 0}(q=0)=-\frac{e^{2}}{\pi \epsilon_{0}\left(\epsilon_{r}+1\right)} \ln \left(e^{1-\gamma} 2^{-1} \frac{\xi}{L}\right)
$$

where $\gamma$ denotes Euler's constant. Combining both limits from Eqs. (S6) and (S8) through a logarithmic interpolation yields

$$
K=\left[1+\frac{V(q=0)}{\pi \hbar v_{F}}\right]^{-1 / 2}=\left[1-\frac{e^{2}}{\pi^{2} \hbar v_{F} \epsilon_{0}\left(\epsilon_{r}+1\right)} \ln \left(e^{-\frac{1}{2}} 2^{-\epsilon_{r}} \frac{w}{L}+e^{1-\gamma} 2^{-1} \frac{\xi}{L}\right)\right]^{-1 / 2} .
$$


This final result only deviates negligibly from a numerical evaluation of the full expression (S4). A theoretical estimate of $K$ in $\mathrm{HgTe} / \mathrm{CdTe} \mathrm{QWs}$ was performed in the work by Teo and Kane [S3], who employ screening by a metallic gate in the vicinity of the edge channel and obtain a similar logarithmic interpolation formula. Using the experimental parameters $v_{\mathrm{F}}=5.3 \times 10^{5} \mathrm{~m} \mathrm{~s}^{-1}$ and $\xi=30 \mathrm{~nm}$, they estimate the Luttinger parameter as $K \approx 0.8$, which indicates rather weak interactions. In contrast, we restrict ourselves to a dielectric screening by the SiC substrate, that keeps the long range character of the Coulomb interaction intact. The logarithmic divergence of the electrostatic energy of the edge channel in the limit of $L \rightarrow \infty$ reflects the incomplete screening of the long-ranged Coulomb interaction. Our result is consistent with the observation that $K$ vanishes for infinitely long systems if metallic screening is neglected. S9 Eq. (S9) is therefore only valid if $L$ is small compared to the distance between metallic gates and the edge channel. The explicit estimate for the bismuthene edge mode is obtained using $w=0.3 \mathrm{~nm}, \xi=0.41 \mathrm{~nm}$, $v_{F}=5.5 \times 10^{5} \mathrm{~ms}^{-1}$ as experimental parameters. By assuming a range of $L=10^{-8}-10^{-6} \mathrm{~m}$ for the edge channel length and $\epsilon_{r}=10$ for the relative permittivity of the SiC substrate $\mathrm{S10}$, we obtain a Luttinger parameter of $K=0.5 \pm 0.1$. This places the $1 \mathrm{D}$ edge channel of bismuthene beyond the regime of weak interactions. It should be noted that $\epsilon_{r}=10$ for undoped $\mathrm{SiC}$ is an estimate in this simple modeling, as $\epsilon_{r}$ is known to increase for heavily doped semiconductors S11, which would result in enhanced screening. Notwithstanding further possible refinements of the current theoretical analysis, our approximation puts the theoretical prediction within the range of the Luttinger parameter extracted from STS scaling in experiment (see Tab. I). Overall, the central parameter that governs the strong decrease in $\mathrm{K}$ from $\mathrm{HgTe} / \mathrm{CdTe} \mathrm{QWs}$ to bismuthene is the reduced channel width $\xi$, which implies stronger Coulomb interactions induced by a substantially increased confinement of the quasi-1D DOS of the helical edge mode. 


\section{STS spectra in close-up analysis}

a) Logarithmic plot

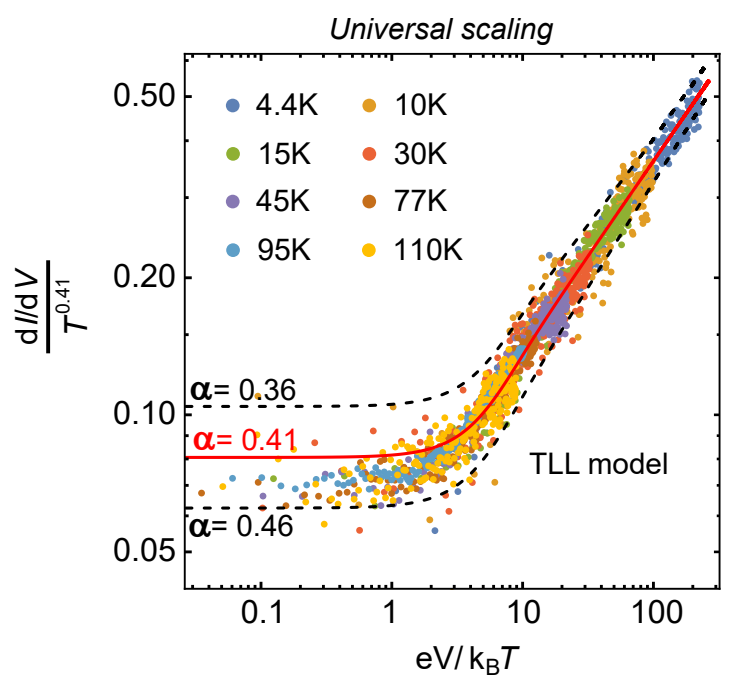

FIG. S3. Universal scaling of the ZBA: hallmark of a TLL. Double-logarithmic plot of the rescaled ZBA spectra of Fig. 4. All spectra collapse onto a single universal curve predicted as a hallmark for tunneling into a TLL. Red curve: $\rho_{\mathrm{TLL}}$ according to Eq. (1) (taking into account thermal and instrumental broadening) with exponent $\alpha=0.41$. Black dashed curve: for \pm 0.05 variation of $\alpha$ to test statistical confidence.

\section{b) Set-point variation}

It is known that the electric field of the tunneling tip can induce band bending effects if the tunneling current is too high and the tip-to-sample distance $z$ becomes to small. We excluded possible tip induced effects on the power-law scaling of the ZBA by measuring spectra for different set-point voltages and currents ranging from $-0.1 \mathrm{~V} \leq V_{\text {set }} \leq$ $-0.3 \mathrm{~V}$ and $0.01 \mathrm{nA} \leq I_{\text {set }} \leq 1.0 \mathrm{nA}$ which apart from an overall scaling factor had no significant influence, see Fig. S4.
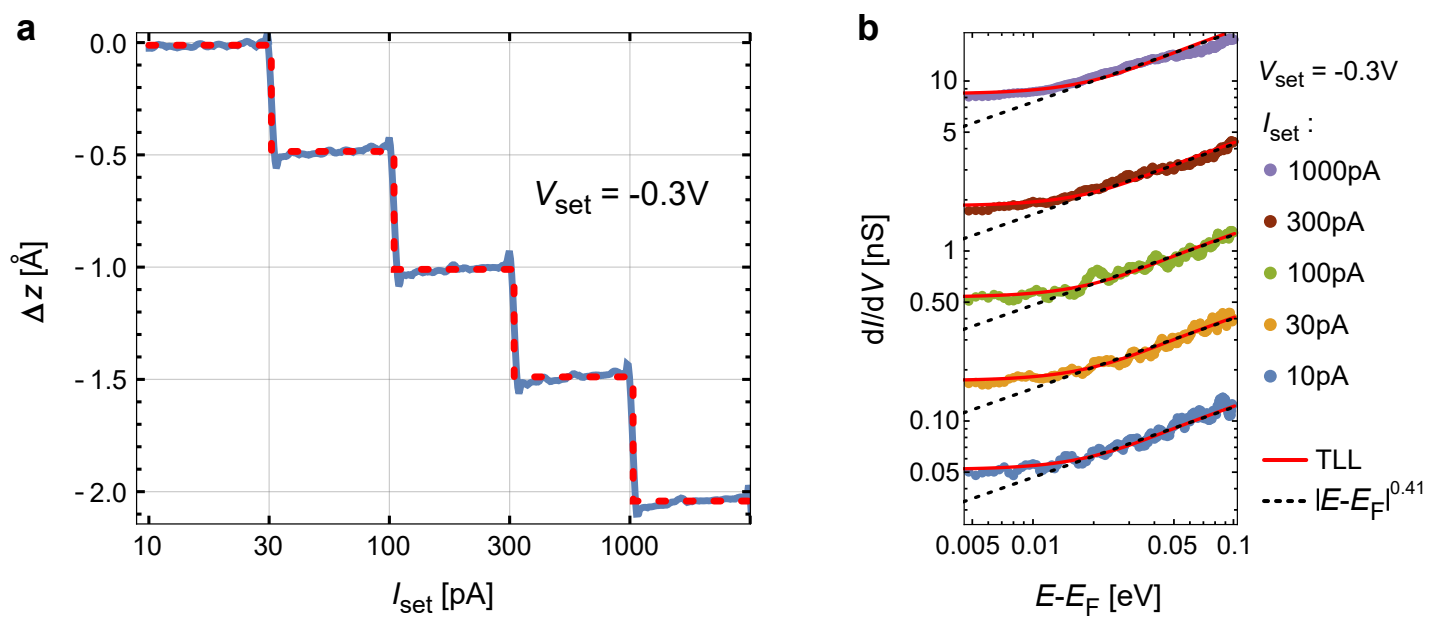

FIG. S4. Influence of $I_{\text {set }}$ on the power-law of the ZBA. a, Tip-to-sample variation $\Delta z$ as a function of the set-point current $I_{\text {set }}$ at fixed set-point bias voltage $V_{\text {set }}=-0.3 \mathrm{~V}$ and at a fixed bismuthene edge position. This nicely reflects that the tunneling current depends exponentially on the tip-to-sample distance. b. Double-logarithmic plot of the ZBA at $T=77 \mathrm{~K}$ measured for different set-point currents $I_{\text {set }}$ and therefore also the tip-to-sample distance according to a. Black dashed line: power-law dependence $d I / d V \sim\left|E-E_{\mathrm{F}}\right|^{0.41}$. Red line: TLL model according to Eq. (1) (taking into account thermal and instrumental broadening). 


\section{Alternative Models for a Zero Bias Anomaly}

The characteristic feature of a TLL is the power-law decay of the LDOS at low-energies. Moreover, $d I / d V(V)$ spectra for different temperatures collapse onto an universal scaling curve as in Fig. 4 and S3. Yet, there are also alternative mechanisms that could potentially lead to a ZBA.

\section{a) Efros-Schklovskii like Coulomb Pseudogap}

In a low-dimensional metallic system the presence of disorder can lead to a suppression of spectral weight around zero energy. The phenomenon is an example for an Efros-Shklovskii like Coulomb pseudogap. The expected LDOS can be found to exhibit an exponential suppression according to [S12]

$$
\begin{aligned}
\rho(V, T)= & \rho_{0} \operatorname{coth}\left(\frac{e V}{2 k_{B} T}\right) 2 T \int_{0}^{\infty} \frac{\sin \left(e V t / k_{B}\right) \cos \left(\sqrt{\left.2 e \Omega t / k_{B}\right)}\right.}{\sinh (\pi t T)} \times \\
& \times \exp \left[-\sqrt{\frac{\Omega}{\pi}} \int_{0}^{\infty} \frac{1-\cos \left(V^{\prime} t\right)}{V^{\prime 3 / 2} \tanh \left(e V^{\prime} / 2 k_{B} T\right)} d V^{\prime}\right] d t
\end{aligned}
$$

where $\Omega:=f_{0}^{2} / 32 \pi D_{0}$ depends on intrinsic properties of the system only, i.e., the electron-electron interaction $f_{0}$ and the diffusion coefficient $D_{0}$, which reflects the strength of disorder in the system. The parameter $\Omega$ will serve as a fitting parameter to our experimental data.

In contrast to the TLL model, however, the functional form of the arising ZBA in this scenario is described by an exponential decrease of the $d I / d V$ for low-energies. The functional form of the Coulomb pseudogap model according to Eq. (S11) with the interaction strength $\Omega$ as the only free parameter is shown in Fig. S5. Whereas a coarse approximation to the data can be achieved for higher sample bias, only poor accordance is given for lower sample bias around $V=0$. Particularly, matching of the exponential form according to Eq. (S11) with the data can not be achieved over the entire bias range of the ZBA. This evidences that a disorder induced Efros-Schklovskii-like Coulomb pseudogap cannot explain the observed spectral behavior.

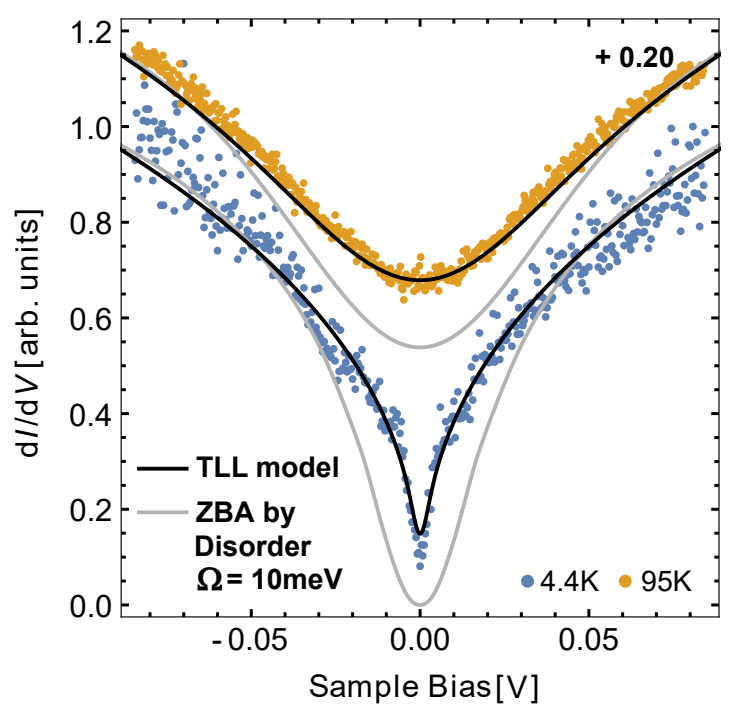

FIG. S5. Comparison with the disorder induced ZBA model. The measured ZBA in bismuthene at $4.36 \mathrm{~K}$ (orange dots) and at $95 \mathrm{~K}$ (blue dots) is compared with the expected $d I / d V(V)$ spectrum from the TLL model (dashed black line) and the model for a disorder induced ZBA model (gray line). The spectra were normalized at $V=100$ meV and the spectrum taken at $95 \mathrm{~K}$ is shifted by 0.20 . In the case of the disorder induced ZBA close matching can not be achieved over the whole energy range. A coarse approximation to the data can be achieved at higher binding energies, but the match is increasingly poor at lower energies around zero-bias. On the other hand, the TLL model reproduces the spectrum over the entire energy range with $\alpha=0.41$. 
In case that the applied bias voltage $V$ is smaller than the resulting charging energy of the system, i.e., $e V<E_{C}=$ $e^{2} / C_{\Sigma}$ where $C_{\Sigma}$ is the total capacitance (described in the following), charging effects can in principle induce a ZBA in STS measurements. As an important example, one may consider an electrical circuit that contains an electromagnetic environment (e.g., the substrate) modeled by external impedances in the tunneling circuit. This type of models are usually referred to as dynamical Coulomb blockade (DCB) models.

In the framework of the DCB scenario, the STS tunneling experiment can conveniently be regarded as a double junction as in Fig. S6 [S13, S14. Electrons tunnel from a metallic tip onto an 'island' on the sample surface and subsequently into the substrate. Part of this double junction is a first tunneling junction (T-junction) which models the tunneling between tip and island with an effective capacitance $C_{T}$ in parallel with a resistance $R_{T}$. The tunneling resistance $R_{T}$ can be inferred from the set-point current and voltage as $R_{T}=V_{\text {set }} / I_{\text {set }}$, and is typically 0.1 G $\Omega \lesssim$ $R_{T} \lesssim 10 \mathrm{G} \Omega$ in our experiments. The tunneling capacitance $C_{T}$ is usually not known, but typically on the order of $C_{T} \lesssim 1 \mathrm{aF}$. [S13, S14] The second junction which models a tunneling process of electrons from the sample surface into the substrate is a second $R C$-junction (S-junction) in series with the T-junction. The resistance $R$ and capacitance $C$ of the S-junction are usually unknown and form fit parameters in the DCB model.

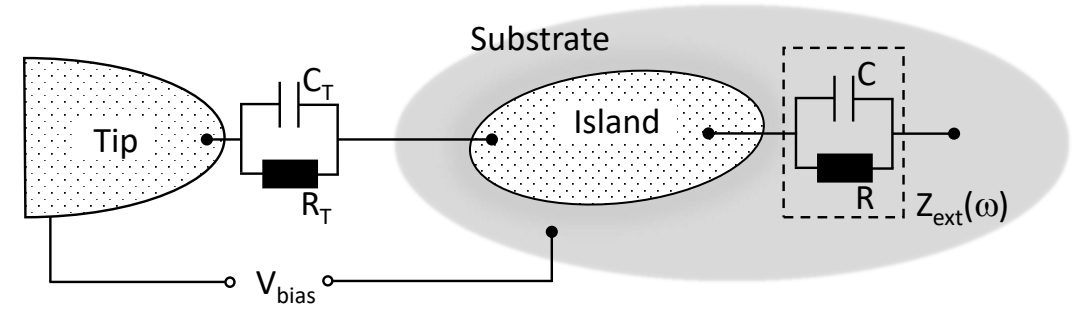

FIG. S6. Model for dynamical Coulomb blockade Schematic picture of electron tunneling between a metallic source and drain through an intermediate island coupled to a dissipative environment $Z_{\text {ext }}(\omega)$. Tunneling can be blocked if the electrostatic energy $E_{C}$ of a single excess electron on the island is large compared to the energy scale of thermal fluctuations.

The following model in terms of the DCB scenario is valid, if in an experimental STS measurement the complete bias voltage drops over the T-junction, i.e., $R_{T} \gg R$, and as long as $R$ is comparable to the resistance quantum $R_{K}=25.8 \mathrm{k} \Omega$. Then tunneling in the DCB regime along with an excitation of the dissipative environment $Z_{\text {ext }}(\omega)$ can be treated quantum mechanically by the so-called environment-quantum fluctuation or $P(E)$-theory [S15], within which the tip to sample (sample to tip) tunneling probability $\Gamma^{+}\left(\Gamma^{-}\right)$at temperature $T$ can be calculated as

$$
\begin{aligned}
& \Gamma^{+}=\frac{1}{e^{2} R_{T}} \int_{-\infty}^{\infty} \int_{-\infty}^{\infty} \rho_{t}(E) \rho_{s}\left(E^{\prime}-e V\right) f(E, T)\left[1-f\left(E^{\prime}-e V, T\right)\right] P\left(E-E^{\prime}\right) d E d E^{\prime} \\
& \Gamma^{-}=\frac{1}{e^{2} R_{T}} \int_{-\infty}^{\infty} \int_{-\infty}^{\infty} \rho_{t}(E) \rho_{s}\left(E^{\prime}-e V\right)[1-f(E, T)] f\left(E^{\prime}-e V, T\right) P\left(E^{\prime}-E\right) d E d E^{\prime},
\end{aligned}
$$

where $V$ is the bias voltage, $f$ is the Fermi function and $E\left(E^{\prime}\right)$ are the energy levels at the tip (sample), respectively. $\rho_{t}$ and $\rho_{s}$ are the tip and sample LDOS, respectively. $P\left(E-E^{\prime}\right)$ describes the probability that the electron looses the energy $E-E^{\prime}$ to the dissipative environment during the tunneling process. In the case of elastic tunneling where energy is conserved within the double junction during the tunneling event the probability factor $P\left(E-E^{\prime}\right)$ reduces to a delta function $P\left(E-E^{\prime}\right)=\delta\left(E-E^{\prime}\right)$.

In principle, the $P(E)$ function can be calculated as [S15]:

$$
P(E)=\frac{1}{2 \pi \hbar} \int_{-\infty}^{\infty} \exp [J(t)+i E t / \hbar] d t
$$

with

$$
J(t)=2 \int_{0}^{\infty} \frac{\operatorname{Re}[Z(\omega)]}{\omega R_{K}} \frac{e^{-i \omega t}-1}{1-e^{-\hbar \omega / k_{B} T}} d \omega,
$$

where $Z(\omega)=\left[i \omega C_{T}+Z_{\text {ext }}^{-1}(\omega)\right]^{-1}$ is the frequency dependent complex impedance as seen from the tunnel junction $\left[\mathrm{S} 13\right.$. The frequency dependent complex impedance of the dissipative environment $Z_{\text {ext }}(\omega)=\left[i \omega C+R^{-1}\right]^{-1}$, 
on the other hand, depends solely on $R$ and $C$ of the S-junction. Consequently, the total impedance can be written as $Z(\omega)=\left[i \omega C_{\Sigma}+R^{-1}\right]^{-1}$, with $C_{\Sigma}=C_{T}+C$. The total tunneling current is calculated as the sum of the tunneling current in both directions $I(V)=-e\left(\Gamma^{+}-\Gamma^{-}\right)$.

In the following calculations, $\rho_{t}$ and $\rho_{s}$ are both taken to be constant in the bias range $-100 \mathrm{mV}<V<100 \mathrm{mV}$, which is reasonable since the tip has been ensured to be metallic and it has been seen that the bismuthene edge LDOS inside the bulk gap, despite the ZBA, is approximately constant. The functional form of the differential conductivity can be calculated as:

$$
\frac{d I}{d V}(V)=\frac{1}{R_{T}}\left[1+2 \int_{0}^{\infty} \frac{\pi k_{B}^{2} T^{2}}{\hbar^{2}} \operatorname{Im}\left[e^{J(t)}\right] \cos \left(\frac{e V t}{\hbar}\right) \operatorname{csch}^{2}\left(\frac{\pi k_{B} T t}{\hbar}\right) t d t\right]
$$

In the case of $T \rightarrow 0$ and $V \rightarrow 0$ the $I(V)$-characteristic and the differential conductance $d I / d V(V)$ become $[$ S15]

$$
\begin{aligned}
I(V) & =\frac{\exp (-2 \gamma / g)}{\Gamma(2+2 / g)} \frac{V}{R_{T}}\left(\frac{\pi e|V|}{g E_{C}}\right)^{2 / g} \\
& \rightarrow \frac{d I}{d V}(V) \sim|V|^{2 / g}
\end{aligned}
$$

It is apparent that in this limit the $d I / d V(V)$ is also characterized by a power-law (with a power-law exponent $\left.2 / g=2 R / R_{K}\right)$, as is the case for tunneling into a TLL. This fact was pointed out by Safi et al. [S16].

For arbitrary temperatures, however, it is not possible to give an analytic solution of the $d I / d V(V)$. One has to calculate it numerically by solving Eq. (S14). In Fig. S7 we present the result for different values of $R$ and $C_{\Sigma}$ and plot it on a rescaled conductivity $(d I / d V) \mathrm{T}^{-2 \mathrm{R} / \mathrm{R}_{\mathrm{K}}}$ and energy scale $e V\left(k_{B} T\right)^{-1}$ in order to detect coincidental universal scaling behavior in the bias voltage range $-100 \mathrm{mV}<V<100 \mathrm{mV}$ (the voltage range where we observed the ZBA in bismuthene measurements). In the DCB model all ZBAs collapse onto a universal scaling curve only for parameters $R=0.2 R_{K}$ and $C_{\Sigma}=0.7 \mathrm{aF}$ and for spectra in the temperature range $4 \mathrm{~K}<T<110 \mathrm{~K}$. In general, whereas $C_{\Sigma}=C_{T}+C$ mainly determines the depth and width of the ZBA, the main effect of $R$ is to determine the steepness of the ZBA.

We now want to discuss implications for our ZBA measurements that follow from the DCB model. First, we want to note that within the DCB model the metallic bismuthene edge channels form the capacitive island and are thought to have poor electrical contact to the $\mathrm{SiC}$ substrate modeled by the S-junction. A crude estimate is that bismuthene edge channels which cover a smaller area $A$ on the $\mathrm{SiC}$ substrate will affect the charging of the S-junction and consequently enlarge $R$ and reduce $C$ according to: $C \propto A=d \times l$ and $R \propto A^{-1}=(d \times l)^{-1}$ for a straight metallic edge with length $l$ and width $d$ (typically $d \lesssim 1.5 \mathrm{~nm}$, see Fig. 1(c)). Therefore, $C$ and $R$ are local properties of the system [S14]. Local deviations should therefore manifest in local differences of the power-law exponent $2 / g$ and more importantly impede universal scaling. We measured the ZBA on four different samples and on various positions on each sample. The observation that we did not measure any significant deviations of the power-law exponent, not even for local variations of $E_{\mathrm{F}}(\gtrsim 100 \mathrm{meV})$, the size of the bismuthene domain nor variations of the set-point parameters $I_{\text {set }}$ and $V_{\text {set }}$ (Fig. S4), together with universal scaling (Fig. 4 and S3), strongly points towards the ZBA being a result of intrinsic properties of the $1 \mathrm{D}$ edge channel and hence the exclusion of the DCB scenario. 


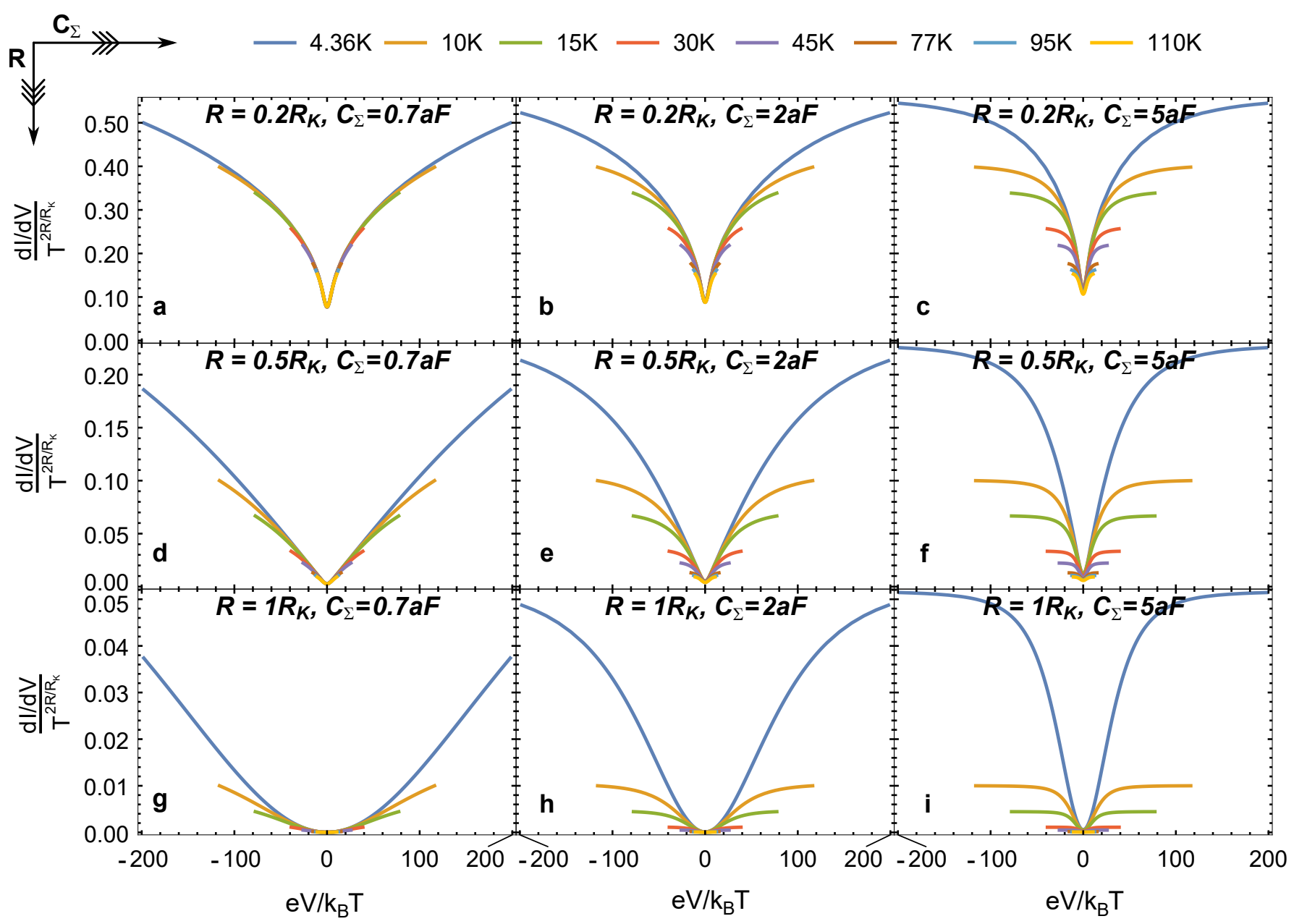

FIG. S7. Scaling of the ZBA in the DCB model. Numerical solution of Eq. S14 for different values of $R$ and $C_{\Sigma}=C_{T}+C$ plotted on a rescaled conductivity (according to $(d I / d V) T^{-2 R / R_{K}}$ ) and energy scale (according to $\left.e V\left(k_{B} T\right)^{-1}\right)$. In general, whereas $C_{\Sigma}$ mainly determines the depth and width of the ZBA, the main effect of $R$ is to determine the steepness of the ZBA. In the energy range $-100 \mathrm{mV}<V<100 \mathrm{mV}$ where we observe the ZBA in our measurements a coincidentally occurring universal scaling law, as in the case of a TLL, is only observed for $R=0.2 R_{K}$ and $C_{\Sigma}=0.7$ aF, where all ZBAs collapse onto a universal scaling curve for temperatures $4.4 \mathrm{~K} \leq T \leq 110 \mathrm{~K}$. 
[S1] T. Li, P. Wang, H. Fu, L. Du, K. A. Schreiber, X. Mu, X. Liu, G. Sullivan, G. A. Csáthy, X. Lin, and R.-R. Du, Phys. Rev. Lett. 115, $136804(2015)$.

[S2] J. I. Väyrynen, F. Geissler, and L. I. Glazman, Phys. Rev. B 93, 241301 (2016)

[S3] J. C. Y. Teo and C. L. Kane, Phys. Rev. B 79, 235321 (2009)

[S4] S. Eggert, Phys. Rev. Lett. 84, 4413 (2000).

[S5] C. Blumenstein, J. Schäfer, S. Mietke, S. Meyer, A. Dollinger, M. Lochner, X. Y. Cui, L. Patthey, R. Matzdorf, and R. Claessen, Nat Phys 7, 776 (2011)

[S6] M. Bockrath, D. H. Cobden, J. Lu, A. G. Rinzler, R. E. Smalley, L. Balents, and P. L. McEuen, Nature 397, 598 (1999)

[S7] A. Calzona, M. Carrega, G. Dolcetto, and M. Sassetti, Phys. Rev. B 92, 195414 (2015).

[S8] T. Giamarchi, Quantum Physics in One Dimension, Clarendon Press Oxford (2003).

[S9] H. J. Schulz, Mesoscopic Quantum Physics, Proceedings of the Les Houches Summer School, Session LXI, Elsevier Publishers (1994).

[S10] L. Patrick and W. J. Choyke, Phys. Rev. B 2, 2255 (1970).

[S11] S. Dhar and A. H. Marshak, Solid-state Electronics 28, 763 (1985).

[S12] L. Bartosch and P. Kopietz, The European Physical Journal B - Condensed Matter and Complex Systems 28, 29 (2002).

[S13] C. Brun, K. H. Müller, I.-P. Hong, F. Patthey, C. Flindt, and W.-D. Schneider, Phys. Rev. Lett. 108, 126802 (2012).

[S14] F. Ming, T. S. Smith, S. Johnston, P. C. Snijders, and H. H. Weitering, PRB 97, 075403 (2018).

[S15] M. H. Devoret, D. Esteve, H. Grabert, G.-L. Ingold, H. Pothier, and C. Urbina, Phys. Rev. Lett. 64, 1824 (1990)

[S16] I. Safi and H. Saleur, Phys. Rev. Lett. 93, 126602 (2004). 